\title{
Heurísticas para o Problema de Dimensionamento de Lotes com Máquinas Paralelas Flexíveis
}

\author{
M. C. F. CATELAN ${ }^{1}$, S. A. DE ARAUJO ${ }^{2}$, D. J. FIOROTTO ${ }^{3 *}$ e D. M. CARVALHO ${ }^{4}$ \\ Recebido em 23 de fevereiro de 2019 / Aceito em 14 de abril de 2020
}

\begin{abstract}
RESUMO. Este artigo trata do problema de dimensionamento de lotes com máquinas paralelas flexíveis, que consiste basicamente em determinar a quantidade de itens a serem produzidos, em cada uma das máquinas, em um horizonte de tempo finito, satisfazendo uma demanda pré-estabelecida. Este problema tem origem econômica e envolve custos de produção, estoque e preparação de máquinas. No problema padrão, cada item pode ser produzido em qualquer uma das máquinas, ou seja, tem-se a flexibilidade total de máquinas. No entanto, nem sempre é viável ter flexibilidade total das máquinas, devido aos altos custos relacionados. Portanto, pode ser de grande importância implementar apenas uma flexibilidade limitada das máquinas. A consideração ou não da flexibilidade de uma máquina para produzir um produto específico torna-se uma variável de decisão e há um custo de investimento associado a estas decisões. Uma formulação matemática para esse problema foi proposta na literatura e os resultados computacionais obtidos por meio de um pacote de otimização combinatória mostraram que resolvê-la pode ser uma tarefa difícil, especialmente para instâncias com muitos itens. Sendo assim, neste trabalho foram propostas três heurísticas para o problema, com intuito de buscar boas soluções em baixos tempos computacionais. Experimentos foram realizados para comparar as heurísticas com a resolução do modelo via pacote de otimização. Pôde-se observar que conforme o número de itens e de máquinas foram aumentando, as soluções com melhor qualidade foram obtidas por apenas uma das heurísticas. Em termos de tempos computacionais, todas as heurísticas obtiveram em média tempos inferiores aos do CPLEX.
\end{abstract}

Palavras-chave: problema de dimensionamento de lotes, flexibilidade de máquinas, heurísticas.

\section{INTRODUÇÃ̃O}

Empresas vêm considerando a possibilidade de investir em um planejamento que trata da produção, aquisição de recursos e matérias-primas. O objetivo é manter a qualidade e baixar os

\footnotetext{
*Autor correspondente: Diego Jacinto Fiorotto - E-mail: diego.fiorotto@ fca.unicamp.br

${ }^{1}$ Departamento de Matemática Aplicada, IBILCE, Universidade Estadual Paulista, R. Cristóvão Colombo, 2265, 15054000, São José do Rio Preto, SP, Brasil - E-mail: melka_cfc@ hotmail.com https://orcid.org/0000-0003-0241-4058

${ }^{2}$ Departamento de Matemática Aplicada, IBILCE, Universidade Estadual Paulista, R. Cristóvão Colombo, 2265, 15054000, São José do Rio Preto, SP, Brasil - E-mail: silvio.araujo@ unesp.br https://orcid.org/0000-0002-4762-2048

${ }^{3}$ Faculdade de Ciências Aplicadas - FCA/UNICAMP, R. Pedro Zaccaria, 1300, 13484-350, Limeira, SP,Brasil - E-mail: diego.fiorotto@fca.unicamp.br https://orcid.org/0000-0002-9594-2716

${ }^{4}$ Departamento de Matemática Aplicada, IBILCE, Universidade Estadual Paulista, R. Cristóvão Colombo, 2265, 15054000, São José do Rio Preto, SP, Brasil - E-mail: desymcb@gmail.com https://orcid.org/0000-0001-9999-1032
} 
custos, buscando o plano mais econômico. O investimento em planejamento de produção pode ser um diferencial e fazer com que empresas se sobressaiam sobre as outras.

O problema de dimensionamento de lotes consiste, basicamente, em determinar a quantidade de itens a ser produzida em cada período de um horizonte de tempo finito. A produção dos itens pode ser realizada por uma ou várias máquinas e deve satisfazer restrições de recursos que estão disponíveis e a uma demanda a ser atendida. Esse problema tem origem econômica e envolve custos de produção, estoque e preparação de máquinas.

O problema estudado neste trabalho enfoca o planejamento da produção, em máquinas paralelas com capacidade limitada. Além disso, considera-se a possibilidade de investir em flexibilidade baseando-se nas ideias de Fiorotto et al. [4] que apresentam uma formulação para o problema em questão.

Em geral, o dimensionamento de lotes é uma competência tática e operacional que interpreta as decisões de planejamento estratégico e as transforma em planos de produção concretos. Enquanto a flexibilidade é uma competência estratégica, na qual empresas podem usá-la para ajustar com precisão o número de máquinas, produtos, serviços e processos. Note que, em alguns casos, há uma integração entre ambas as decisões, que podem ser tomadas simultaneamente. Uma aplicação de decisões de flexibilidade neste contexto pode ser encontrada, por exemplo, na indústria de semicondutores, onde uma receita tem que ser qualificada em uma máquina específica, o que pode ser caro e demorado $[7,11,16]$. Portanto, nem todas as receitas são qualificadas para todas as máquinas. No entanto, para lidar com as alterações do produto, o processo de qualificação pode ser reconsiderado periodicamente. Em nosso contexto, considera-se que as decisões de flexibilidade são tomadas no início do horizonte de planejamento e então permanecem fixas para o restante do horizonte.

Na prática, é difícil tomar decisões sobre flexibilidade, pois o número de preparação para a produção de um produto cresce exponencialmente de acordo com o número de itens e máquinas e, além disso, é difícil calcular seus benefícios. Observe que considerar uma flexibilidade total de máquinas pode ser muito caro, principalmente se os itens são muito diferentes, o que torna importante a tarefa de estudar maneiras de implementar uma flexibilidade de tal forma que os custos e os lucros sejam equilibrados.

Na literatura estudada, com exceção de Fiorotto et al. [4], não foram encontrados estudos que determinam os benefícios da flexibilidade no contexto de dimensionamento de lotes. Estudos em outros contextos e que consideram um ponto de vista estocástico como, por exemplo, estudos sobre decisões estratégicas em ambientes estocásticos podem ser encontrados em Fine e Freund [3], Jordan e Graves [12] e Tang e Denardo [19].

As principais contribuições deste artigo são: desenvolver três heurísticas de solução com o intuito de determinar as ligações entre máquinas e itens, ou seja, determinar quais máquinas podem produzir cada um dos itens, com a finalidade de gerar boas soluções em baixos tempos computacionais; Comparar em um extensivo estudo computacional os resultados obtidos pelo solver 
CPLEX v. 12.9 ao resolver a formulação matemática do problema alvo deste artigo, baseada na modelagem proposta por Fiorotto et al. [4], com os resultados obtidos pelas três heurísticas.

As outras seções deste trabalho estão divididas da seguinte maneira: Na Seção 2 são apresentados estudos sobre flexibilidade e uma formulação para o problema de dimensionamento de lotes com máquinas paralelas flexíveis. Na Seção 3 propõem-se três heurísticas com o intuito de obter boas soluções com baixos tempos computacionais. Na Seção 4 apresentam-se os resultados computacionais e na Seção 5 são realizadas as conclusões e apresentadas ideias para trabalhos futuros.

\section{TRABALHOS CORRELATOS E MODELAGEM MATEMÁTICA}

Nesta seção apresentam-se os estudos relacionados a flexibilidade de máquinas, bem como, uma formulação baseada na proposta por Fiorotto et al. [4].

A Figura 1 mostra um exemplo com 6 itens, 6 máquinas e 4 configurações diferentes de flexibilidade. Note que a Figura 1.(a) mostra o caso dedicado, onde cada item está sendo produzido em apenas uma máquina específica. As Figuras 1.(b) e 1.(c) contém o mesmo número de ligações entre itens e máquinas (mesma quantidade de flexibilidade), no entanto, estas ligações foram adicionadas de maneira distinta. Na Figura 1.(b) tem-se 3 grupos com 2 máquinas e 2 itens e na Figura 1.(c) tem-se a configuração de cadeia longa, em que todas máquinas e itens são interligados. Na Figura 1.(d) tem-se a flexibilidade total, onde todos os itens estão conectados a todas as máquinas.

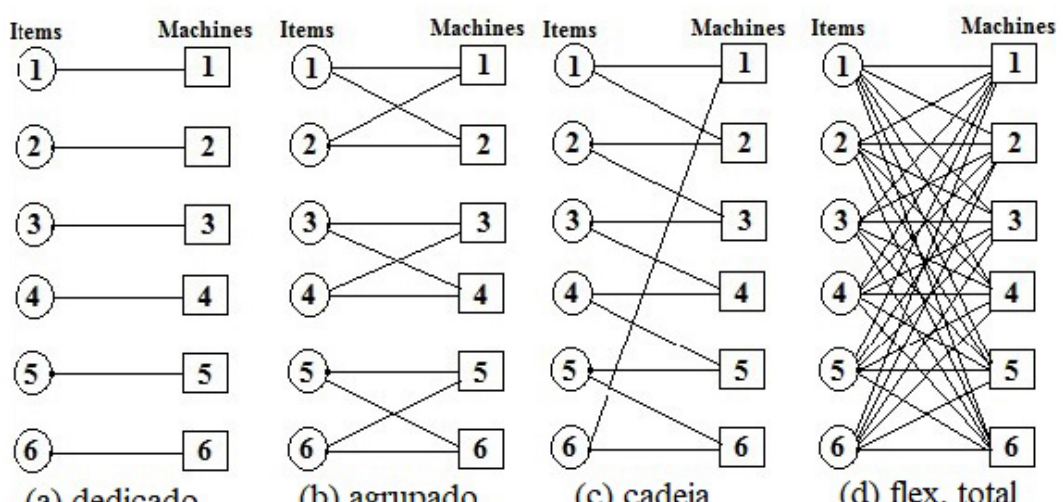

(a) dedicado

(b) agrupado

(c) cadeia

(d) flex. total

Figura 1: Diferentes configurações de flexibilidade com 6 itens e 6 máquinas. Imagem retirada de [4].

Jordan e Graves [12] foram os primeiros a considerar a concepção e a eficácia de uma quantidade limitada da flexibilidade de máquinas. Eles analisaram o valor da flexibilidade no processo de fabricação em um modelo estocástico com um único período e um único estágio de produção, no qual várias máquinas são capacitadas para produzir vários produtos diferentes, de forma que 
cada máquina pode ser dedicada a um único produto ou flexível para produzir vários produtos distintos. Como a demanda é aleatória e a capacidade é limitada, o aumento de flexibilidade faz com que a quantidade produzida aumente. Desta forma, a quantidade de itens em atraso pode ser reduzida, diminuindo os custos totais de produção. Em [12] compreende-se que quase todos os benefícios da flexibilidade podem ser alcançados implementando apenas uma pequena quantidade de flexibilidade, mas de uma maneira inteligente. Os autores referem-se a este como o princípio da cadeia, Figura 1.(c). Uma "cadeia" define-se por um grupo de itens e máquinas que estão todos conectados, direta ou indiretamente, por decisões de atribuição de produto. Dentro de uma cadeia, um caminho pode ser traçado entre qualquer item e máquina através das ligações de atribuição do produto. A ideia-chave por trás do encadeamento é que o excesso de capacidade pode ser deslocado ao longo da cadeia. Este artigo abriu novos caminhos para linhas de pesquisa relacionadas ao estudo de flexibilidade. Na literatura sobre sistemas de produção, ainda é uma das estratégias mais influentes que é estudada e utilizada na prática.

Diversos autores estudaram os benefícios da flexibilidade em um ambiente de produção estocástico. Sheikhzadeh et al. [17] apresentaram modelos analíticos para a avaliação do desempenho do princípio da cadeia em relação a flexibilidade total. Graves e Tomlin [5] estenderam o trabalho de Jordan e Graves [12] para cadeias de fornecimento de múltiplos estágios e desenvolveram ideias sobre estratégias para a implementação da flexibilidade do processo. Inman et al. [8] mostraram como aplicar o princípio da cadeia ao treinamento cruzado em linhas de montagem. Iravani et al. [9] demonstraram que usar a aplicação de treinamento cruzado em sistemas com um menor número de ligações pode superar os sistemas com maior número de ligações. Muriel et al. [14] desenvolveram modelos analíticos para estudar o impacto da flexibilidade sobre a escassez e estoques de componentes para sistemas de manufatura sob encomenda de múltiplos produtos com várias plantas. Mak e Shen [13] ressaltaram que é fundamental avaliar a compensação entre o custo do investimento em flexibilidade e os benefícios resultantes. Eles mostram que a heurística de encadeamento, fortemente defendida, às vezes pode ter um desempenho insatisfatório quando os recursos não são perfeitamente flexíveis. Simchi-Levi e Wei [18] apresentaram um estudo teórico sobre a efetividade do princípio da cadeia.

Embora vários trabalhos tenham mostrado que o princípio da cadeia tem um desempenho muito bom, especialmente para sistemas homogêneos (ou seja, com baixa variabilidade nos dados), há dois trabalhos que mostram que nem sempre isso é válido. Gurumurthi e Benjaafar [6] apresentaram resultados numéricos para sistemas de filas (que consiste num sistema heterogêneo), mostrando que, neste caso, há sempre uma configuração não encadeada que funciona melhor que o encadeamento. Andradóttir et al. [1] reforçam que o encadeamento é desejável em ambientes homogêneos e indicam que outras configurações de flexibilidade geralmente apresentam melhor desempenho em ambientes heterogêneos.

Ainda que o problema de dimensionamento de lotes com máquinas paralelas, com uma quantidade limitada de flexibilidade seja uma extensão natural do pressuposto padrão, e torna o problema mais realista com o que acontece na prática, existem poucos trabalhos que abordam esta extensão. Em uma aplicação na indústria de pneus, Jans e Degraeve [10] discutiram um problema 
em que nem todos os tipos de pneus podem ser produzidos em todas as máquinas. Xiao [21] propõem uma heurística baseada em hibridização Lagrangiana e recozimento simulado para o problema de escalonamento de lotes em máquinas paralelas capacitado, em que nem todas as máquinas são elegíveis para produzir todos os itens. Além disso, consideram que para cada item existe uma máquina de preferência, aumentando a qualidade dos itens e a confiabilidade da máquina.

\subsection{Formulação com Flexibilidade de Máquina como uma Variável de Decisão}

Nesta seção é apresentada uma modelagem matemática que se baseia na formulação clássica do problema de dimensionamento de lotes com máquinas paralelas, com a possibilidade de haver atrasos (backorder) e flexibilidade de máquina, proposta por Fiorotto et al. [4]. O investimento de atualizar uma máquina para um produto específico é uma variável de decisão do problema e há um orçamento global sobre as decisões de investimento.

Para a formulação matemática do problema considere os seguintes parâmetros:

$\begin{array}{lll}I & : & \text { conjunto de itens, } I=\{1, \ldots, n\} ; \\ J & : & \text { conjunto de máquinas, } J=\{1, \ldots, m\} ; \\ T & : & \text { conjunto de períodos, } T=\{1, \ldots, p\} ; \\ d_{i t} & : & \text { demanda do item } i \text { no período } t ; \\ s d_{i t \tau} & : & \text { soma da demanda para o item } i, \text { do período } t \text { até o período } \tau(\tau \geq t) ; \\ h c_{i t} & : & \text { custo unitário de estoque do item } i \text { no período } t ; \\ b c_{i t} & : & \text { custo unitário de atraso do item } i \text { no período } t ; \\ s c_{i j t} & : & \text { custo de preparação do item } i \text { na máquina } j \text { no período } t ; \\ v c_{i j t} & : & \text { custo de produção do item } i \text { na máquina } j \text { no período } t ; \\ s t_{i j t} & : & \text { tempo de preparação do item } i \text { na máquina } j \text { no período } t ; \\ v t_{i j t} & : \text { tempo de produção do item } i \text { na máquina } j \text { no período } t ; \\ f c_{i j} & : \text { custo de flexibilidade para produzir o item } i \text { na máquina } j ; \\ F m a x & : \text { orçamento para investir em flexibilidade; } \\ \text { Cap }_{j t} & : \text { capacidade (em unidade de tempo) da máquina } j \text { no período } t .\end{array}$

As variáveis de decisão são definidas por:

$x_{i j t} \quad: \quad$ quantidade produzida do item $i$ na máquina $j$ no período $t$;

$y_{i j t}$ : variável binária, indicando a produção (1) ou não (0) do item $i$ na máquina $j$ no período $t$;

$s_{i t} \quad: \quad$ quantidade de estoque do item $i$ no final do período $t$;

$b_{i t} \quad: \quad$ quantidade de atraso do item $i$ no final do período $t$;

$z_{i j} \quad$ : variável binária que recebe o valor 1 se a máquina $j$ pode produzir o item $i$ e 0 , caso contrário; 
Desta forma, a formulação do problema é dada por:

$$
\min \sum_{j \in J} \sum_{i \in I} \sum_{t \in T}\left(s c_{i j t} y_{i j t}+v c_{i j t} x_{i j t}\right)+\sum_{i \in I} \sum_{t \in T}\left(h c_{i t} s_{i t}+b c_{i t} b_{i t}\right)
$$

Sujeito a:

$$
\begin{array}{lr}
s_{i, t-1}-b_{i, t-1}+\sum_{j \in J} x_{i j t}=d_{i t}+s_{i t}-b_{i t} & \forall i \in I, t \in T \\
x_{i j t} \leq \min \left\{\left(\operatorname{Cap}_{j t}-s t_{i j t}\right) / v t_{i j t}, s d_{i 1 p}\right\} y_{i j t} & \forall j \in J, i \in I_{j}, t \in T \\
\sum_{i \in I}\left(s t_{i j t} y_{i j t}+v t_{i j t} x_{i j t}\right) \leq \operatorname{Cap}_{j t} & \forall j \in J, t \in T \\
y_{i j t} \leq z_{i j} & \forall j \in J, i \in I, t \in T \\
\sum_{i \in I} \sum_{j \in J} f c_{i j} z_{i j} \leq F \max & \forall i \in I \\
z_{i i}=1 & \forall j \in J, i \in I_{j}, t \in T \\
y_{i j t} \in\{0,1\}, z_{i j} \in\{0,1\}, x_{i j t} \geq 0 & \forall i \in I, t \in T \\
s_{i t} \geq 0, s_{i 0}=0, s_{i p}=0, b_{i t} \geq 0, b_{i 0}=0 & \forall
\end{array}
$$

A função objetivo (2.1) minimiza os custos de preparação, produção, estoque e de atrasos. As restrições (2.2) garantem o equilíbrio do estoque em cada período considerando a possibilidade de atrasos. A seguir estão as restrições de preparação da máquina (2.3) e o limite de capacidade (2.4). A fim de tornar a formulação mais forte, limitamos a produção para cada item na restrição (2.3) tanto pela soma da demanda quanto pelo limite máximo possível com a capacidade disponível. As restrições (2.5) garantem que uma máquina possa ser preparada para produzir um item somente se esta máquina tem a flexibilidade para produzir este item. A restrição (2.6) limita o orçamento para o investimento em flexibilidade. As restrições (2.7) obrigam que as máquinas tenham flexibilidade para a configuração dedicada em que toda máquina $i$ produz exclusivamente o item $i, i \in I$. Neste trabalho, consideraremos que a quantidade de máquinas é igual à quantidade de itens produzidos. Finalmente, (2.8) e (2.9) definem os domínios das variáveis.

\section{MÉTODOS DE SOLUÇÃO}

Nessa seção serão apresentadas as três heurísticas desenvolvidas para resolver o problema de dimensionamento de lotes com máquinas paralelas flexíveis (2.1) - (2.9). Observa-se que em Fiorotto et al. [4], considerando o número de links igual $2 n$ e a versão 12.6 do CPLEX, o tempo médio para resolver as instâncias com 24 itens e 24 máquinas foi de 8988,91 segundos, com um gap médio de $11,5 \%$.

A ideia de propor as heurísticas surgiu depois de vários experimentos computacionais que mostraram as vantagens e desvantagens de usar um software de otimização como o CPLEX v. 12.9. Apesar do elevado tempo computacional gasto para obter soluções para instâncias de médio a grande porte (12 e 24 itens), o CPLEX 12.9 obteve um bom desempenho para resolver instâncias 
de pequeno porte (6 itens) e algumas de médio porte (12 itens). Tendo isso em vista, as três heurísticas propostas nas próximas seções utilizam tal ferramenta para resolver problemas menores com o intuito de acelerar o processo para encontrar uma solução de boa qualidade em baixo tempo computacional.

Os passos das heurísticas serão descritos a seguir. Observe que, assim como em Fiorotto et al. [4], na restrição (2.6) fixam-se os custos de flexibilidade $f c_{i j}$ em 1 e $F$ max no número de ligações que são permitidas para serem adicionadas. Assim, limita-se o número total de ligações que podem ser utilizadas, isto é, impõem-se um limite no número de combinações possíveis entre máquinas e itens.

\subsection{Primeira Heurística (HEUR-I)}

A primeira heurística, chamada aqui de HEUR-I, se assemelha a um método fix-and-optimize [15] uma vez que, a cada iteração, parte das variáveis de decisão é fixada e o restante é otimizado. $\mathrm{O}$ método de solução da HEUR-I pode ser descrito da seguinte maneira:

\section{- Passo 1: Definir parâmetros iniciais.}

Sejam $v$, um parâmetro correspondente a iteração atual do algoritmo, e $F_{v}$ o orçamento para investir em flexibilidade (ligações permitidas) na iteração $v$. Inicialmente, fazem-se $v=1$ e $F_{v}=n+1$ como apresentado nas linhas 2 e 3, respectivamente, do Algoritmo 1.

\section{- Passo 2: Determinar o plano de produção com até $F_{v}$ ligações.}

Resolve-se o problema (3.1) - (3.4) (linha 5 do Algoritmo 1);

$$
\min \sum_{j \in J} \sum_{i \in I} \sum_{t \in T}\left(s c_{i j t} y_{i j t}+v c_{i j t} x_{i j t}\right)+\sum_{i \in I} \sum_{t \in T}\left(h c_{i t} s_{i t}+b c_{i t} b_{i t}\right)
$$

Sujeito a:

$$
\begin{array}{ll}
\text { Restrições }(2.2)-(2.5),(2.7)-(2.9) & \\
\sum_{i \in I} \sum_{j \in J} f c_{i j} z_{i j} \leq F_{v} & \\
z_{i j}=1 & \forall\left\{(i, j) \mid i \neq j \text { e } z_{i j}^{v-1}=1\right\}
\end{array}
$$

As restrições (3.4) são responsáveis por fixarem novas ligações tendo em vista a solução encontrada na solução anterior representada por $\left(x^{\nu-1}, y^{v-1}, z^{v-1}, s^{v-1}, b^{v-1}\right)$. Quando $v=$ 1 , considera-se que $z_{i j}^{v-1}=0$ para todo $\operatorname{par}(i, j)$, em que $i \neq j$.

\section{- Passo 3: Atualizar $v$ e $F_{v}$.}

Após resolver o problema (3.1)-(3.4), atualizam-se $v$ e $F_{v}$ fazendo $v=v+1$ e $F_{v}=F_{v-1}+1$, respectivamente (linhas 6 e 7 do Algoritmo 1). 


\section{- Passo 4: Verificar condição de parada.}

Os Passos 2 e 3 são realizados enquanto $F_{v} \leq F \max$ (linhas 4-8 do Algoritmo 1). Se $F_{v}>$ Fmax, o método retorna a solução encontrada (linha 10 do Algoritmo 1).

Um pseudo-código da heurística HEUR-I é apresentado no Algoritmo 1.

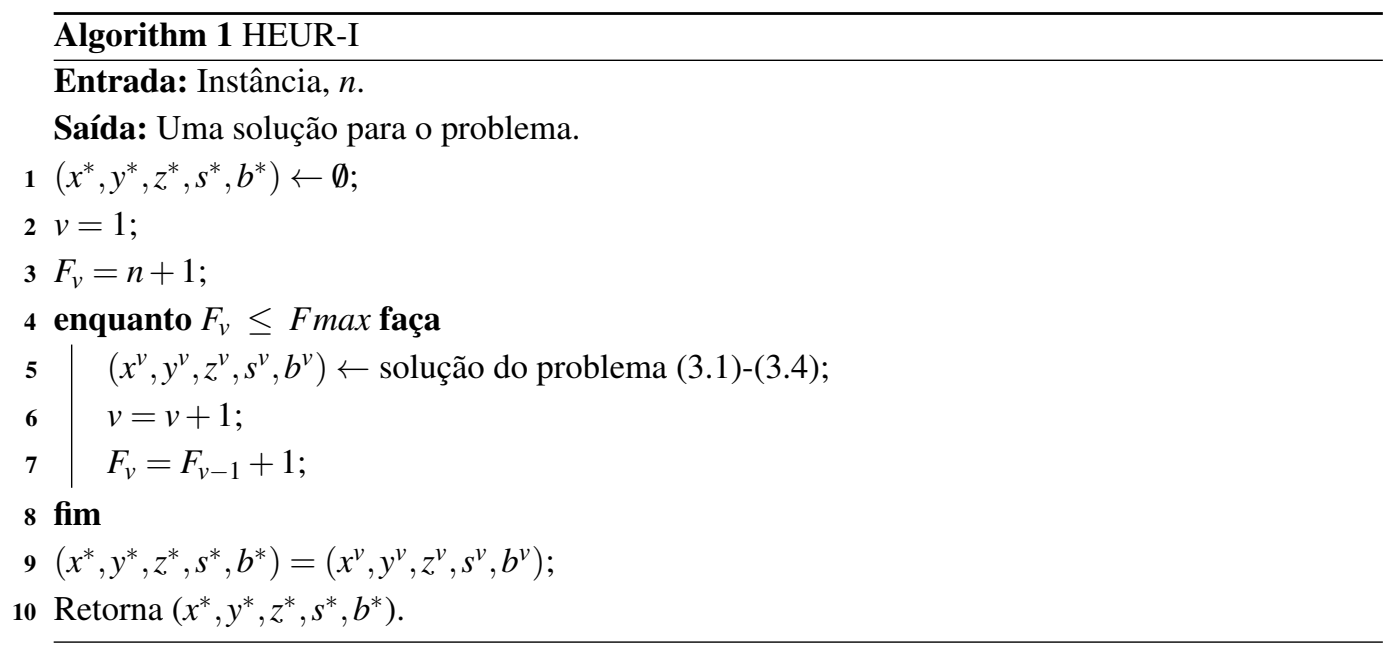

\subsection{Segunda Heurística (HEUR-II)}

Assim como a HEUR-I, a segunda heurística proposta, denotada por HEUR-II, realiza um processo semelhante ao de uma heurística fix-and-optimize. A diferença da HEUR-I para a HEUR-II está na forma como uma nova ligação é adicionada à configuração de flexibilidade das máquinas. Sendo assim, a HEUR-II determina, a cada iteração do método, as ligações $\left(z_{i j}\right)$ entre os itens e as máquinas que serão adicionados utilizando para a escolha do item aquele com o maior custo de atraso na solução atual. Para escolher a máquina estimam-se as economias na solução realizadas com a adição de cada uma das máquinas e escolhe-se aquela com a maior economia.

A estratégia HEUR-II pode ser descrita por meio dos seguintes passos:

\section{- Passo 1: Definir parâmetros.}

Sejam $v$, um parâmetro correspondente a iteração atual do algoritmo, e $F_{v}$ o orçamento para investir em flexibilidade (ligações permitidas) na iteração $v$. Inicialmente, fazem-se $v=1$ e $F_{v}=n+1$ como apresentado nas linhas 2 e 3, respectivamente, do Algoritmo 2. Além disso, utiliza-se um conjunto $S$, inicialmente vazio, para manter o registro das ligações permitidas (linha 1 do Algoritmo 2). Cada ligação escolhida em uma iteração $v$ é representada pelo par de índices $\left(i_{v}, j_{v}\right)$ correspondente ao item e máquina, respectivamente.

- Passo 2: Determinar o plano de produção com $F_{v}$ ligações. 
Resolve-se o problema (3.5) - (3.9) (linha 5 do Algoritmo 2);

$$
\min \sum_{j \in J} \sum_{i \in I} \sum_{t \in T}\left(s c_{i j t} y_{i j t}+v c_{i j t} x_{i j t}\right)+\sum_{i \in I} \sum_{t \in T}\left(h c_{i t} s_{i t}+b c_{i t} b_{i t}\right)
$$

Sujeito a:

$$
\begin{aligned}
& \text { Restrições }(2.2)-(2.5),(2.7)-(2.9) \\
& \sum_{i \in I} \sum_{j \in J} f c_{i j} z_{i j} \leq F_{v} \\
& z_{i j}=1 \\
& z_{i j}=0
\end{aligned}
$$

No problema (3.5) - (3.9), as restrições (3.8) e (3.9) fixam os valores das variáveis que pertencem a solução atual e das que não pertencem, respectivamente. A solução deste problema será representada por $\left(x^{v}, y^{v}, z^{v}, s^{v}, b^{v}\right)$.

\section{- Passo 3: Escolher $i_{v}$.}

Para determinar qual ligação será escolhida para fazer parte da solução atual, verifica-se se há algum item com atraso (linha 6 do Algoritmo 2). Em caso afirmativo, escolhe-se o item com o maior custo total de atraso na solução atual, denotado por $i_{v}$, de acordo com a Equação (3.10).

$$
i_{v}=\left\{i \mid \max _{i \in I}\left(\sum_{t=1}^{p} b c_{i t} b_{i t}^{v}\right)\right\}
$$

\section{- Passo 4: Escolher $j_{v}$.}

Para a escolha da máquina $j_{v}$ correspondente a nova ligação estimam-se as economias obtidas pela flexibilização de cada uma das máquinas para produzir o item $i_{v}$. A máquina que conduz à maior economia é selecionada (linha 7 do Algoritmo 2). Os detalhes para a escolha de $j_{v}$ são apresentados na Seção 3.2.1.

- Passo 5: Atualizar o conjunto $S$ e os parâmetros $v$ e $F_{v}$.

Atualizam-se o conjunto $S$ e os parâmetros $v$ e $F_{v}$ da seguinte maneira: $S=S \cup\left\{\left(i_{v}, j_{v}\right)\right\}$, $v=v+1$ e $F_{v}=F_{v-1}+1$, linhas 8,9 e 10 , respectivamente, do Algoritmo 2.

\section{- Passo 6: Verificar condição de parada.}

Os passo 2 a 5 (linhas 4-11 do Algoritmo 2) são realizados até que todas as Fmax ligações tenham sido inseridas na solução. Se $F_{v}>F \max$ o método retorna a solução encontrada (linha 13 do Algoritmo 2).

Um pseudo-código da HEUR-II é apresentado no Algoritmo 2. 


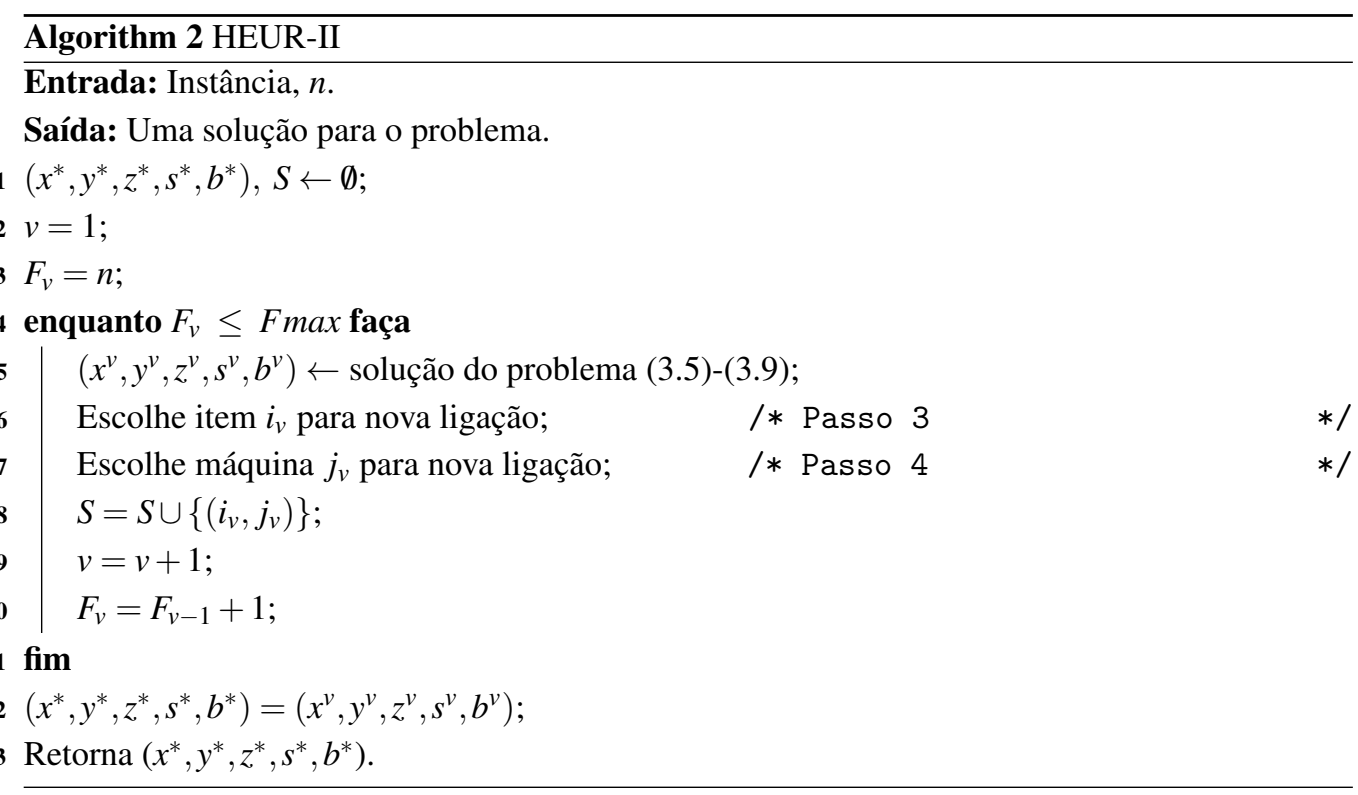

\subsubsection{Estimativa de economia}

A estimativa das economias geradas pela inclusão de uma ligação não é uma tarefa simples, haja vista que essa estimativa depende de vários fatores, como por exemplo, os custos de produção, a capacidade atualizada das máquinas, os atrasos de cada item, entre outros. Tendo em vista a formulação (2.1) - (2.9), note que não é possível determinar a quantidade exata de atraso por meio das variáveis $x_{i j t}$. Isso acontece porque essas variáveis determinam a quantidade produzida dos itens, mas não em quais períodos essas quantidades vão ser utilizadas. A Tabela 3 ilustra esse fato para um exemplo com um item e três períodos. Observe que no período 2 tem-se uma produção de 9 unidades e não há informação se parte desta produção é utilizada para satisfazer as 5 unidades de atraso do período 1 (e assim criar 3 unidades de atraso no período 2) ou se essa produção será utilizada para satisfazer toda demanda do período 2 e apenas parte do atraso do período 1 .

Tabela 3: Exemplo.

\begin{tabular}{lccc}
\hline período & 1 & 2 & 3 \\
\hline demanda & 10 & 7 & 5 \\
produção & 5 & 9 & 8 \\
\hline
\end{tabular}

Para determinar a quantidade aproximada de itens em atraso por período $\left(\right.$ atraso $\left._{i t}\right)$, supõe-se que, se a produção de um período é maior do que a demanda, utilizase a produção para satisfazer a demanda do período em questão e então abre-se a possibilidade 
de satisfazer itens em atraso. Sendo assim, quando $t>1 \mathrm{e}\left(\left(\sum_{j=1}^{m} x_{i j t}+s_{i t-1}\right)<d_{i t}\right)$, atraso $_{i t}$ é calculado como na Equação (3.11).

$$
\operatorname{atraso}_{i t}=d_{i t}-\sum_{j=1}^{m} x_{i j t}+\text { atraso }_{i t-1}-s_{i t-1}
$$

Se $t=1$ e $\sum_{j=1}^{m} x_{i j t}<d_{i t}$, calcula-se atraso $_{i t}$ como mostra a Equação (3.12).

$$
\operatorname{atraso}_{i 1}=d_{i 1}-\sum_{j=1}^{m} x_{i j 1}
$$

Desta forma, a quantidade de atraso de cada período do exemplo apresentado na Tabela (3) é encontrado da seguinte maneira:

$$
\begin{aligned}
& \operatorname{atraso}_{1}=10-5=5 \\
& \operatorname{atraso}_{2}=0, \text { pois }\left(\sum_{j=1}^{m} x_{t}+s_{t-1}\right)>d_{t} \\
& \operatorname{atraso}_{3}=0, \text { pois }\left(\sum_{j=1}^{m} x_{t}+s_{t-1}\right)>d_{t}
\end{aligned}
$$

Logo, o atraso total $\left(\sum_{t=1}^{p}\right.$ atraso $\left._{t}\right)$ é de 5 unidades a partir do período 1.

Para calcular uma estimativa das economias, primeiro, calculam-se a capacidade mínima total aproximada $\left(\alpha_{i j}\right)$ para produzir o item em atraso e a capacidade disponível $\left(\right.$ folga $\left._{j}\right)$ de cada máquina como apresentado nas Equações (3.13) e (3.14), respectivamente.

$$
\begin{aligned}
\alpha_{i j} & =\sum_{t=1}^{p}\left(\text { atraso }_{i t} v t_{i j t}+\frac{\text { atraso }_{i t}}{Q_{j t}} s t_{i j t}\right) \\
\text { folga }_{j} & =\sum_{t=1}^{p}\left(\text { Cap }_{j t}-\sum_{i=1}^{n}\left(v t_{i j t} x_{i j t}+s t_{i j t} y_{i j t}\right)\right)
\end{aligned}
$$

Em que, $Q_{j t}:=\frac{\operatorname{Cap}_{j t}-s t_{i j t}}{v t_{i j t}}$ é a quantidade máxima aproximada que a máquina $j$ pode produzir do item $i$ no período $t$.

Em seguida, calcula-se uma estimativa das economias (beneficio ${ }_{i j}$ ) produzidas por cada uma das máquinas de acordo com os valores de $\alpha_{i j}$ e $f o l g a_{j}$. Se a capacidade disponível de uma máquina for maior do que a capacidade total necessária para resolver o atraso, ou seja, $\alpha_{i j} \leq f o l g a_{j}$, o custo total de atraso do item $i$ pode ser eliminado. Portanto, o benefício é dado pelo custo total de atraso menos o custo total para produzir a quantidade de itens em atrasos como apresentado na Equação (3.15). 


$$
\text { beneficio }_{i j}=\sum_{t=1}^{p}\left(b c_{i t} b_{i t}-\text { atraso }_{i t} v c_{i j t}-\frac{\text { atraso }_{i t}}{Q_{j t}} s c_{i j t}\right)
$$

Por outro lado, se a capacidade disponível for menor do que a capacidade total necessária para resolver o atraso $\left(\alpha_{i j}>f o l g a_{j}\right)$, pode-se eliminar apenas parte do custo de atraso. Seja $P_{j t}:=$ $\left(\frac{f o l g a_{j}-\frac{f o l g a_{j}}{\text { Cap }_{j t}} s t_{i j t}}{v t_{i j t}}\right)$ a quantidade total aproximada de itens que podem ser produzidos com a capacidade disponível, o benefício será dado pela Equação (3.16).

$$
\text { beneficio }_{i j}=\sum_{t=1}^{p}\left(P_{j t}\left(b c_{i t}-v c_{i j t}\right)-\frac{P_{j t}}{Q_{j t}}\right)
$$

Após calcular as economias aproximadas (bene ficio $_{i j}$ ) obtidas ao adicionar uma ligação em todas as máquinas, escolhe-se o par $(i, j)$ com maior benefício.

\subsection{Terceira Heurística (HEUR-III)}

Assim como em HEUR-II, a escolha do item da HEUR-III é realizada de acordo com o maior custo de atraso na solução do problema linearmente relaxado. No entanto, para selecionar a máquina optou-se por explorar a aproximação da relaxação linear das variáveis $z_{i j}$. Caso não exista item com atraso, a escolha da nova ligação também leva em consideração os valores das variáveis do problema linearmente relaxado. A estratégia HEUR-III, portanto, utiliza ideias de uma heurística relax-and-fix uma vez que, a cada iteração, se baseia na solução do problema relaxado para definir quais variáveis fixar.

A heurística pode ser descrita da seguinte maneira:

\section{- Passo 1: Definir parâmetros.}

Sejam $v$, um parâmetro correspondente a iteração atual do algoritmo, e $F_{v}$ o orçamento para investir em flexibilidade (ligações permitidas) na iteração $v$. Inicialmente, fazem-se $v=1$ e $F_{v}=n+1$ como apresentado nas linhas 2 e 3, respectivamente, do Algoritmo 3. Além disso, utiliza-se um conjunto $S$, inicialmente vazio, para manter o registro das ligações permitidas (linha 1 do Algoritmo 3). Cada ligação escolhida em uma iteração $v$ é representada pelo par de índices $\left(i_{v}, j_{v}\right)$ correspondente ao item e máquina, respectivamente.

\section{- Passo 2: Determinar o plano de produção com até $F_{v}$ ligações.}

Resolve-se o seguinte problema relaxado (linha 5 do Algoritmo 3):

$$
\min \sum_{j \in J} \sum_{i \in I} \sum_{t \in T}\left(s c_{i j t} y_{i j t}+v c_{i j t} x_{i j t}\right)+\sum_{i \in I} \sum_{t \in T}\left(h c_{i t} s_{i t}+b c_{i t} b_{i t}\right)
$$


Sujeito a:

$$
\begin{array}{lr}
\text { Restrições }(2.2)-(2.5),(2.7) \text { e (2.9) } \\
\begin{array}{lr}
\sum_{i \in I} \sum_{j \in J} f c_{i j} z_{i j} \leq F_{v} & \\
z_{i j}=1 & \forall \quad(i, j) \in S \\
y_{i j t} \in[0,1], z_{i j} \in[0,1], x_{i j t} \geq 0 & \forall j \in J, i \in I_{j}, t \in T
\end{array}
\end{array}
$$

As restrições (3.21) definem o domínio relaxado das variáveis y e $z$. Considere $\left(x^{v}, y^{v}, z^{v}, s^{v}, b^{v}\right)$ a representação da solução do problema (3.17)-(3.21).

\section{- Passo 3: Escolher $i_{v}$.}

Para determinar qual ligação será escolhida para fazer parte da solução atual, verifica-se se há algum item com atraso (linha 6 do Algoritmo 3). Em caso afirmativo, escolhe-se o item com o maior custo total de atraso na solução atual, denotado por $i_{v}$, de acordo com a Equação (3.22).

$$
i_{v}=\left\{i \mid \max _{i \in I}\left(\sum_{t=1}^{p} b c_{i t} b_{i t}^{v}\right)\right\}
$$

Caso não haja itens em atraso, analisa-se todos os valores relaxados das variáveis $z_{i j}^{v}$. Escolhe-se a variável com maior valor e atribui-se à $i_{v}$ e $j_{v}$ seus respectivos índices $i$ e $j$ como apresentado na Equação (3.23).

$$
\left(i_{v}, j_{v}\right)=\left\{(i, j) \mid \max _{i \in I, j \in J}\left(z_{i j}^{v}\right)\right\}
$$

Se existir algum item em atraso, o método segue para o Passo 4 (linha 6 do Algoritmo 3), caso contrário, executa-se o Passo 5 (linhas 7-9 do Algoritmo 3). Deve-se observar que foi testada a possibilidade de fixar mais de uma variável por iteração, mas tal estratégia não surtiu efeito nos resultados.

\section{- Passo 4: Selecionar ligação.}

Escolhe-se a máquina $j$ com o maior valor da variável relaxada $z_{i_{v} j}^{v}$ com o item $i_{v}$, definido no Passo 3, para ser $j_{v}$ como mostra a Equação (3.24).

$$
j_{v}=\left\{j \mid \max _{j \in J}\left(z_{i_{v} j}^{v}\right)\right\}
$$

Se para todo par $\left(i_{v}, j\right)$, de item e máquina, o valor da variável $z_{i_{v} j}^{v}$ for 0 , analisam-se todos os valores relaxados da variável $z_{i j}^{v}$, para todo $i$ e $j$, e atribui-se a $i_{v}$ e $j_{v}$ os índices da variável com maior valor como na Equação (3.23). 


\section{- Passo 5: Atualizar o conjunto $S$ e os parâmetros $v$ e $F_{v}$.}

Atualizam-se o conjunto $S$ e os parâmetros $v$ e $F_{v}$ da seguinte maneira: $S=S \cup\left\{\left(i_{v}, j_{v}\right)\right\}$, $v=v+1$ e $F_{v}=F_{v-1}+1$ (linhas 7, 8 e 9, respectivamente, do Algoritmo 3).

\section{- Passo 6: Verificar condição de parada e determinar plano de produção final.}

Os Passos 2 a 5 (linhas 4-10 do Algoritmo 3) são realizados até adicionar todas as Fmax ligações. Após adicionar todas as ligações resolve-se o problema (2.1)-(2.9),(3.20), considerando as variáveis $y_{i j t}$ e $z_{i j}$ como binárias (linha 11 do Algoritmo 3). Caso exista uma solução para o problema (2.1)-(2.9),(3.20), retorna-se a solução encontrada (linha 12 do Algoritmo 3).

Um pseudo-código da HEUR-III é apresentado no Algoritmo 3. Note que, assim como em uma heurística relax-anf-fix, o método HEUR-III não garante que uma solução factível seja encontrada.

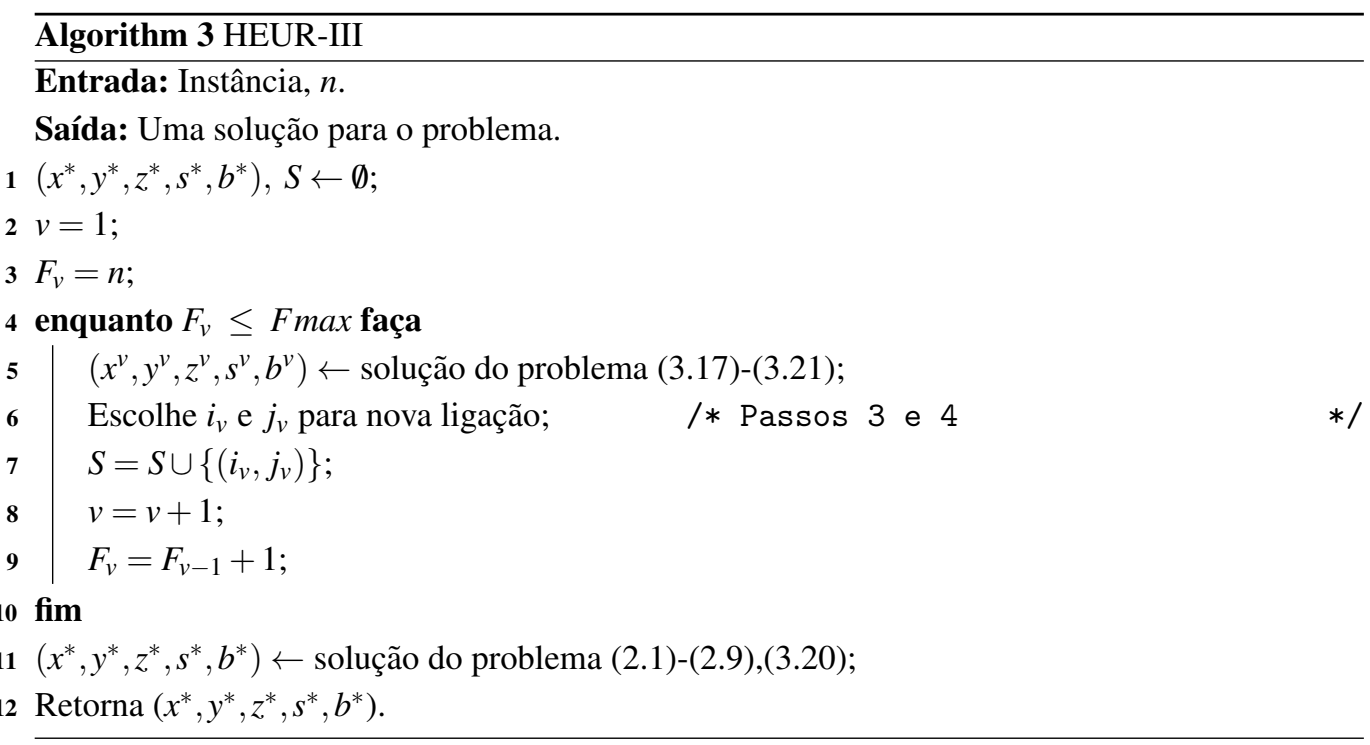

\section{EXPERIMENTOS COMPUTACIONAIS}

Nesta seção são apresentados a geração de dados e os resultados computacionais obtidos neste trabalho. Os resultados mostrados são os obtidos pelo CPLEX v.12.9 ao resolver a formulação (2.1) - (2.9), e pelas três heurísticas propostas na Seção 3.

\subsection{Geração de Dados}

Os dados utilizados neste trabalho são os mesmos utilizados em Fiorotto et al. [4], os quais consistem de uma adaptação dos dados propostos por Trigeiro [20] para o problema de dimensionamento de lotes com uma única máquina. Mais especificamente, foram utilizados os conjuntos 
de instâncias $F 1-F 20$ e $G 51-G 60$. O conjunto $F 1-F 20$ contém 20 instâncias com 6 itens e 15 períodos. O conjunto $G 51$ - G55 é composto por 5 instâncias com 12 itens e 15 períodos, enquanto o conjunto $G 56$ - G60 contém 5 instâncias com 24 itens e 15 períodos. Com base nas 30 instâncias mencionadas anteriormente, 30 novas instâncias foram criadas para atender o problema com máquinas paralelas idênticas. Sendo assim, nas novas instâncias, as capacidades são idênticas e para um determinado item, os tempos e os custos são os mesmos em todas as máquinas.

Com o intuito de explorar situações com diferentes níveis de atraso, para cada uma das 30 instâncias foi criado um conjunto com 12 instâncias em que a capacidade varia de 40 a 150 (unidades de tempo). A escolha da capacidade das máquinas foi baseada em testes preliminares. Sendo assim, o conjunto de instâncias utilizado para os experimentos possui um total de 360 instâncias (12 níveis de capacidade multiplicado por 30 instâncias em cada nível). Os demais parâmetros que são utilizados estão descritos na Tabela 4.

Tabela 4: Parâmetros.

\begin{tabular}{lc}
\hline PARÂMETROS & INTERVALOS \\
\hline Tempo de produção $v t_{i j t}$ & 1 \\
Custo unitário de estoque $h c_{i t}$ & {$[1,5]$} \\
Tempo de preparação $s t_{i j t}$ & {$[10,50]$} \\
Custo de preparação $s c_{i j t}$ & {$[400,1000]$} \\
Custo de produção $v c_{i j t}$ & 0 \\
Demanda $d_{i t}$ & {$[0,180]$} \\
Custo unitário de atraso $b c_{i t}$ & $100 * h c_{i t}$ \\
\hline
\end{tabular}

\subsection{Ferramentas para Análise de Desempenho dos Métodos}

Para analisar os resultados, foram considerados quatro fatores: variação de capacidade (Cap), limitante superior (LS), gap (Gap) e tempo computacional em segundos (T(s)). Observa-se que os limitantes superiores encontrados pelo CPLEX foram fixados em $100 \%$ e a partir desses valores, calculam-se os valores referente as heurísticas, isto é, utiliza-se a fórmula $\left(100 \times \frac{L S_{H E U R}}{L S_{C P L E X}}\right)$. O gap utilizado corresponde a distância entre o limitante superior encontrado pelo método e o limitante inferior (LI) encontrado pelo CPLEX v.12.9 ao resolver o problema (2.1) - (2.9), como apresenta a Equação (4.1).

$$
g a p=100 \times \frac{L S-L I}{L S}
$$

Além dos fatores mencionados anteriormente, optou-se pela utilização da medida de perfil de desempenho proposta por Dolan e Moré [2] para verificar a qualidade das soluções encontradas. Sejam $\Lambda$ um conjunto de $n_{\lambda}$ algoritmos e $\Psi$ composto pelas $n_{\psi}$ instâncias consideradas no ex- 
perimento, o mecanismo de avaliação calcula a taxa de desempenho de um algoritmo $\lambda \in \Lambda$ em relação a uma instância $\psi \in \Psi$, aqui denotada por $\delta_{\lambda \psi}$, de acordo com a Equação (4.2).

$$
\delta_{\lambda \psi}=\frac{t_{\lambda \psi}}{\min \left\{t_{\lambda \psi} \mid \lambda \in \Lambda\right\}}
$$

em que $t_{\lambda \psi}$ é o valor da métrica sob análise, encontrada pelo algoritmo $\lambda$, com $\psi$ como instância de entrada. Nos experimentos, os métodos foram comparados utilizando-se seus valores de função objetivo e tempos computacionais como métrica de avaliação. Dessa maneira, no perfil, o eixo $y$ indica a função de distribuição de probabilidade para a taxa de desempenho de um valor de função objetivo/tempo, também conhecida como perfil de desempenho. De acordo com Dolan e Moré [2], o perfil de desempenho, aqui chamado de $\Theta_{\lambda}(\tau)$, é equivalente à Equação (4.3).

$$
\Theta_{\lambda}(\tau)=\frac{1}{n_{\psi}}\left|\log _{2} \psi \in \Psi: \delta_{\lambda \psi} \leq \tau\right|
$$

Na Equação (4.3), $\tau$, que representa o eixo $x$ do gráfico, corresponde a todas as possíveis taxas de desempenho resultantes da relação entre o conjunto de algoritmos $\Lambda$ e o conjunto de instâncias $\Psi$. Portanto, a Equação (4.3) indica a probabilidade do algoritmo $\lambda$ ter uma taxa de desempenho dentro de um fator $\tau$.

\subsection{Resultados Computacionais}

A formulação (2.1) - (2.9) e as três heurísticas foram implementadas em C ++ usando o concert e o CPLEX 12.9 como pacotes de otimização. Os testes foram feitos em um computador com processador Intel(R) Core(TM) i7-4790, memória RAM 32GB, com sistema operacional Ubuntu.

Os métodos comparados permitem a criação de diferentes configurações de flexibilidade, sempre partindo da configuração dedicada. O número de ligações adicionadas em cada uma destas configurações é determinado pela quantidade de itens e de máquinas da instância em questão. Dessa forma, para o conjunto com $n$ itens foram adicionadas $n$ ligações (além da configuração inicial dedicada), ou seja, em todos os experimentos foi utilizado $F \max =2 n$. Em [4] os autores mostram que os benefícios da configuração de cadeia longa foram os que mais se aproximaram dos obtidos pela configuração de flexibilidade total.

O limite do tempo computacional foi fixado em 60 segundos para resolver cada iteração dos métodos propostos na Seção 3. Desta forma, o limite de tempo computacional imposto para o CPLEX e todos os outros métodos foi de 420 segundos para as instâncias com 6 itens e 6 máquinas, 780 segundos para 12 itens e 12 máquinas e 1500 segundos para 24 itens e 24 máquinas.

Observa-se que os resultados apresentados nas tabelas a seguir correspondem a média aritmética dos resultados obtidos para cada conjunto de instâncias. 
Na Tabela 5 são apresentadas as médias dos limitantes superiores (LS) e dos gaps (Gap) obtidos pelo CPLEX v. 12.9 e pelas três heurísticas propostas HEUR-I, HEUR-II e HEUR-III ao resolverem as instâncias com 6 itens e 6 máquinas.

A Tabela 5 mostra que, em geral, o CPLEX é eficiente em termos de gaps de solução para resolver instâncias com 6 itens e máquinas, principalmente para problemas com capacidade muito apertadas (entre 40 e 100) e folgadas (entre 140 e 150) de forma que os gaps encontrados pelo CPLEX para estas classes não ultrapassam 1\%. No entanto, para problemas com capacidade média (entre 110 e 130) os gaps encontrados são relativamente altos, chegando até a 11,22\%.

Tabela 5: Resultados obtidos para as instâncias com 6 itens, 6 máquinas e 15 períodos.

\begin{tabular}{|c|c|c|c|c|c|c|c|c|}
\hline \multirow[t]{2}{*}{ Cap } & \multicolumn{2}{|c|}{ CPLEX } & \multicolumn{2}{|c|}{ HEUR-I } & \multicolumn{2}{|c|}{ HEUR-II } & \multicolumn{2}{|c|}{ HEUR-III } \\
\hline & $\mathrm{LS}$ & Gap & LS & Gap & LS & Gap & LS & Gap \\
\hline 40 & 100,00 & 0,03 & 100,00 & 0,02 & 100,38 & 0,38 & 100,09 & 0,10 \\
\hline 50 & 100,00 & $\mathbf{0 , 0 7}$ & 100,01 & 0,08 & 100,67 & 0,69 & 100,18 & 0,26 \\
\hline 60 & 100,00 & 0,09 & 100,03 & 0,12 & 100,31 & 0,44 & 100,27 & 0,38 \\
\hline 70 & 100,00 & 0,14 & 100,04 & 0,18 & 100,79 & 1,21 & 100,19 & 0,33 \\
\hline 80 & 100,00 & 0,15 & 100,09 & 0,25 & 100,58 & 0,76 & 100,25 & 0,48 \\
\hline 90 & 100,00 & 0,34 & 100,19 & 0,52 & 100,83 & 1,45 & 100,77 & 1,33 \\
\hline 100 & 100,00 & 0,79 & 100,29 & 1,18 & 101,09 & 2,20 & 101,07 & 2,27 \\
\hline 110 & 100,00 & 2,53 & 100,81 & 3,79 & 100,95 & 3,81 & 105,52 & 10,87 \\
\hline 120 & 100,00 & 11,22 & 101,63 & 13,40 & 102,16 & 15,30 & 115,44 & 35,43 \\
\hline 130 & 100,00 & 5,39 & 101,13 & 6,65 & 102,80 & 7,73 & 102,49 & 8,03 \\
\hline 140 & 100,00 & 0,68 & 100,11 & 0,78 & 100,07 & 0,75 & 100,26 & 0,91 \\
\hline 150 & 100,00 & 0,42 & 100,00 & 0,42 & 100,00 & 0,42 & 100,13 & 0,56 \\
\hline Média & 100,00 & 1,82 & 100,36 & 2,28 & 100,89 & 2,93 & 102,22 & 5,08 \\
\hline
\end{tabular}

Em relação aos tempos computacionais, observa-se na Tabela 6 que todas as heurísticas se mostraram significativamente mais rápidas do que o CPLEX. Nota-se que os tempos atingidos pela heurística HEUR-III são em média 3 vezes menores, se comparados com as heurísticas HEUR-I e HEUR-II. Além disso, na média geral o tempo computacional da HEUR-III é quase 8 vezes menor do que o tempo gasto pelo CPLEX.

Para o limite superior (LS), como esperado para as instâncias pequenas, todas as heurísticas apresentaram um desempenho igual ou pior ao do CPLEX. A estratégia HEUR-I se mostrou competitiva em média, haja vista que a diferença em relação aos resultados encontrados pelo CPLEX é no máximo 2,18\%, no pior dos casos. Um perfil de desempenho em relação aos valores da função objetivo e tempos computacionais são apresentados nas Figuras $2 \mathrm{a}$ e $2 \mathrm{~b}$, respectivamente.

Em relação aos valores da função objetivo encontrados pelos métodos, pode-se observar na Figura 2a que o CPLEX teve o melhor desempenho (88,75\%) para encontrar os melhores valores. Apesar do desempenho inferior das heurísticas, percebe-se que com uma pequena relaxação dos valores, quando $\tau=1,02$, todos os métodos atingem os melhores resultados para pelo menos 
Tabela 6: Tempos gastos para as instâncias com 6 itens, 6 máquinas e 15 períodos.

\begin{tabular}{rrrrr}
\hline Cap & CPLEX & HEUR-I & HEUR-II & HEUR-III \\
\hline 40 & $\mathbf{0 , 0 3}$ & 0,15 & 0,12 & 0,06 \\
50 & 42,49 & $\mathbf{4 , 8 2}$ & 7,39 & 6,69 \\
60 & 13,39 & $\mathbf{3 , 1 9}$ & 5,64 & 4,17 \\
70 & 111,37 & 19,01 & $\mathbf{1 3 , 3 3}$ & 17,85 \\
80 & 191,89 & 40,23 & 38,25 & $\mathbf{2 8 , 8 6}$ \\
90 & 203,53 & 62,69 & 53,02 & $\mathbf{3 7 , 2 5}$ \\
100 & 352,60 & 114,72 & 111,44 & $\mathbf{4 8 , 1 2}$ \\
110 & 408,24 & 179,73 & 181,73 & $\mathbf{5 5 , 5 9}$ \\
120 & 420,00 & 220,83 & 154,26 & $\mathbf{5 5 , 4 6}$ \\
130 & 420,00 & 191,34 & 185,93 & $\mathbf{4 8 , 8 3}$ \\
140 & 365,28 & 155,12 & 221,15 & $\mathbf{2 7 , 5 1}$ \\
150 & 356,17 & 155,78 & 264,20 & $\mathbf{1 9 , 2 6}$ \\
\hline Média & 240,42 & 95,63 & 103,04 & $\mathbf{2 9 , 1 4}$ \\
\hline
\end{tabular}

Figura 2: Perfil de desempenho dos modelos e CPLEX para encontrar solução para as instâncias com 6 itens e 6 máquinas.

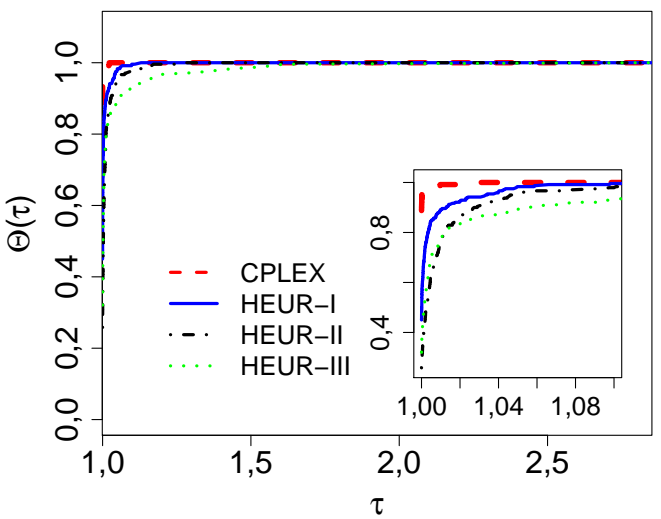

(a) Perfil de desempenho em relação ao valor da função objetivo.

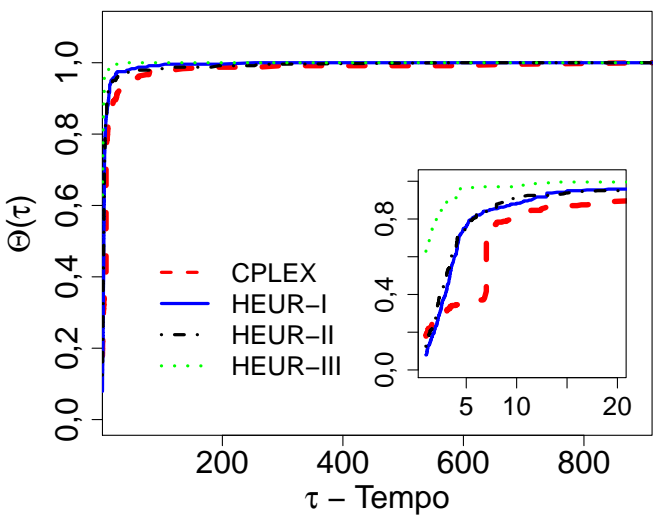

(b) Perfil de desempenho em relação ao tempo.

80\% das instâncias. Em particular, as heurísticas HEUR-I e HEUR-II têm 100\% de chance de atingir os melhores resultados quando $\tau=1,14$ e $\tau=1,28$, respectivamente.

De acordo com a Figura $2 \mathrm{~b}$ pode-se concluir que as heurísticas têm maiores chances de encontrar suas melhores soluções mais rapidamente que o CPLEX, principalmente com a HEUR-III.

A Tabela 7 apresenta as médias dos limitantes superiores (LS) e dos gaps (Gap) para instâncias com 12 itens e 12 máquinas. Para os problemas com capacidade folgada (entre 40 e 90) os 
resultados encontrados pelo CPLEX ainda apresentam valores baixos, como na Tabela 5 os gaps não ultrapassam 1\%. Entretanto, observa-se um aumento significativo dos gaps médios para os problemas com capacidade entre 100 e 140 e com 12 itens quando comparados com as instâncias com 6 itens.

Tabela 7: Resultados obtidos para as instâncias com 12 itens, 12 máquinas e 15 períodos.

\begin{tabular}{|c|c|c|c|c|c|c|c|c|}
\hline \multirow[t]{2}{*}{ Cap } & \multicolumn{2}{|c|}{ CPLEX } & \multicolumn{2}{|c|}{ HEUR-I } & \multicolumn{2}{|c|}{ HEUR-II } & \multicolumn{2}{|c|}{ HEUR-III } \\
\hline & LS & Gap & LS & Gap & LS & Gap & LS & Gap \\
\hline 40 & 100,00 & 0,09 & 100,02 & 0,11 & 100,03 & 0,12 & 100,09 & 0,18 \\
\hline 50 & 100,00 & 0,09 & 100,04 & 0,12 & 100,26 & 0,32 & 100,08 & 0,16 \\
\hline 60 & 100,00 & 0,15 & 100,07 & 0,22 & 100,49 & 0,62 & 100,26 & 0,42 \\
\hline 70 & 100,00 & 0,26 & 100,08 & 0,35 & 100,77 & 1,02 & 100,27 & 0,58 \\
\hline 80 & 100,00 & 0,39 & 100,18 & 0,58 & 100,90 & 1,33 & 100,71 & 1,07 \\
\hline 90 & 100,00 & 1,00 & 100,32 & 1,27 & 102,97 & 3,77 & 100,74 & 1,75 \\
\hline 100 & 100,00 & 1,90 & 101,01 & 2,89 & 105,97 & 6,99 & 102,43 & 4,19 \\
\hline 110 & 100,00 & 6,36 & 99,58 & 6,10 & 102,59 & 8,63 & 104,96 & 10,43 \\
\hline 120 & 100,00 & 26,52 & 96,39 & 24,25 & 108,71 & 32,35 & 103,27 & 28,23 \\
\hline 130 & 100,00 & 16,68 & 96,82 & 14,12 & 107,50 & 21,65 & 98,86 & 16,41 \\
\hline 140 & 100,00 & 2,04 & 99,32 & 1,31 & 100,95 & 2,87 & 106,80 & 7,89 \\
\hline 150 & 100,00 & 0,90 & 100,12 & 1,00 & 100,23 & 1,11 & 106,56 & 6,74 \\
\hline Média & 100,00 & 4,70 & 99,50 & 4,36 & 102,61 & 6,73 & 102,09 & 6,50 \\
\hline
\end{tabular}

Destaca-se que para os problemas com capacidade 120 e 130 os gaps ultrapassam $15 \%$. Em relação aos limitantes superiores, observa-se que apesar das heurísticas HEUR-II e HEUR-III apresentarem resultados um pouco piores dos obtidos pelo CPLEX (102,61\% e 102,09\%, respectivamente, na média), a heurística HEUR-I obteve resultados melhores para 4 dos 12 níveis de capacidade considerados. Além disso, para os outros 8 níveis de capacidade, a HEUR-I atinge uma diferença de no máximo $0,99 \%$ em relação aos limitantes encontrados pelo CPLEX. No geral, a média dos resultados obtidos pela HEUR-I é 0,34\% melhor que a do CPLEX.

Para os tempos computacionais apresentados na Tabela 8, nota-se que o CPLEX utilizou o tempo máximo fixado (780 segundos) para quase todos os níveis de capacidade (70 a 150). Além disso, as heurísticas apresentaram tempos computacionais inferiores quando comparados ao CPLEX de forma que, assim como os resultados apresentados na Tabela 6, o menor tempo computacional é obtido pela heurística HEUR-III.

Uma segunda análise dos resultados leva em consideração o exposto nos perfis de desempenho das Figuras 3a e 3b.

A Figura 3a mostra que apesar das heurísticas terem menos chances de encontrar os melhores valores para a função objetivo (quando $\tau=1$ ), ainda assim as heurísticas se mostram competitivas. A relaxação de $\tau$ para 1,35 é suficiente para que todas as heurísticas tenham $100 \%$ de chances de 
Tabela 8: Tempos gastos para as instâncias com 12 itens, 12 máquinas e 15 períodos.

\begin{tabular}{rrrrr}
\hline Cap & CPLEX & HEUR-I & HEUR-II & HEUR-III \\
\hline 40 & 91,16 & $\mathbf{5 , 5 1}$ & 157,26 & 38,41 \\
50 & 19,05 & $\mathbf{1 7 , 6 3}$ & 202,28 & 39,43 \\
60 & 638,67 & 138,97 & 219,57 & $\mathbf{6 1 , 0 5}$ \\
70 & 780,01 & 258,11 & 355,83 & $\mathbf{5 2 , 9 2}$ \\
80 & 780,01 & 345,88 & 369,10 & $\mathbf{6 1 , 3 5}$ \\
90 & 780,01 & 531,65 & 400,46 & $\mathbf{6 1 , 4 6}$ \\
100 & 780,01 & 598,68 & 495,21 & $\mathbf{6 1 , 6 7}$ \\
110 & 780,01 & 621,79 & 518,74 & $\mathbf{6 1 , 9 3}$ \\
120 & 780,01 & 645,70 & 494,45 & $\mathbf{6 1 , 5 2}$ \\
130 & 780,01 & 686,24 & 513,53 & $\mathbf{5 0 , 1 9}$ \\
140 & 780,01 & 638,50 & 608,35 & $\mathbf{1 4 , 5 8}$ \\
150 & 780,01 & 672,53 & 652,17 & $\mathbf{1 , 4 4}$ \\
\hline Média & 647,41 & 430,10 & 415,58 & $\mathbf{4 7 , 1 6}$ \\
\hline
\end{tabular}

Figura 3: Perfil de desempenho dos modelos e CPLEX para encontrar solução para as instâncias com 12 itens e 12 máquinas.

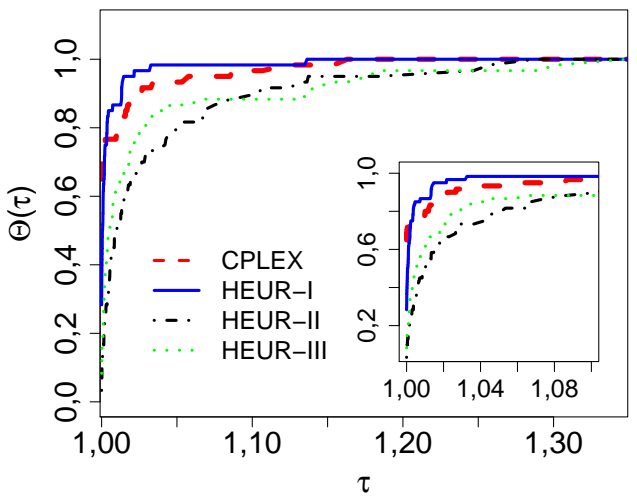

(a) Perfil de desempenho em relação ao valor da função objetivo.

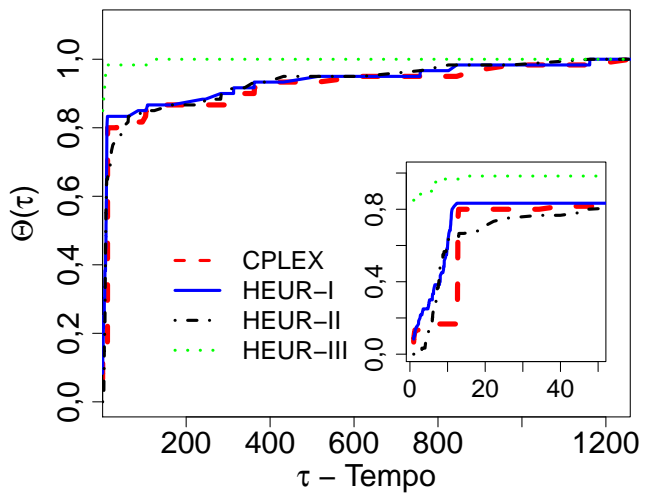

(b) Perfil de desempenho em relação ao tempo.

encontrar os melhores resultados. Em particular, nota-se que quando $\tau \approx 1,01$ a HEUR-I supera o desempenho do CPLEX quando limitado por tempo.

Na Figura 3b observa-se que a HEUR-III tem o melhor desempenho em relação ao tempo consumido para obter seus resultados. A heurística HEUR-I tem um desempenho computacional levemente superior ao CPLEX e a HEUR-II ora tem um desempenho superior, ora tem um desempenho inferior ao do CPLEX. 
Na Tabela 9 são apresentados os resultados para as instâncias grandes, com 24 itens e 24 máquinas. Nota-se que os gaps obtidos com o CPLEX são elevados para vários níveis de capacidade, principalmente para os problemas com capacidade de 100 a 140 . O gap mais alto $(63,13 \%)$ é encontrado na classe com capacidade igual a 130.

Tabela 9: Resultados obtidos para as instâncias com 24 itens, 24 máquinas e 15 períodos.

\begin{tabular}{|c|c|c|c|c|c|c|c|c|}
\hline \multirow[t]{2}{*}{ Cap } & \multicolumn{2}{|c|}{ CPLEX } & \multicolumn{2}{|c|}{ HEUR-I } & \multicolumn{2}{|c|}{ HEUR-II } & \multicolumn{2}{|c|}{ HEUR-III } \\
\hline & $\mathrm{LS}$ & Gap & LS & Gap & LS & Gap & LS & Gap \\
\hline 40 & 100,00 & 0,15 & 100,08 & 0,23 & 100,15 & 0,30 & 100,15 & 0,30 \\
\hline 50 & 100,00 & 0,23 & 100,02 & 0,25 & 101,07 & 1,36 & 102,41 & 2,68 \\
\hline 60 & 100,00 & 0,29 & 99,98 & 0,27 & 103,07 & 3,39 & 106,29 & 6,36 \\
\hline 70 & 100,00 & 0,96 & 99,56 & $\mathbf{0 , 5 0}$ & 104,01 & 4,73 & 128,29 & 21,78 \\
\hline 80 & 100,00 & 1,73 & 99,59 & 1,29 & 121,30 & 17,29 & 178,13 & 44,50 \\
\hline 90 & 100,00 & 3,67 & 98,98 & 2,51 & 117,09 & 17,22 & 254,83 & 44,35 \\
\hline 100 & 100,00 & 7,65 & 102,66 & 9,26 & 238,49 & 60,66 & 404,71 & 69,96 \\
\hline 110 & 100,00 & 22,20 & 87,66 & 13,69 & 216,36 & 62,32 & 904,93 & 85,48 \\
\hline 120 & 100,00 & 49,38 & 76,82 & 33,72 & 228,68 & 67,51 & 2059,42 & 94,02 \\
\hline 130 & 100,00 & 63,13 & 31,18 & 50,05 & 237,54 & 82,55 & 3209,14 & 99,24 \\
\hline 140 & 100,00 & 5,00 & 108,55 & 11,33 & 124,79 & 22,09 & 437,95 & 33,84 \\
\hline 150 & 100,00 & 1,14 & 100,03 & 1,17 & 103,62 & 4,69 & 118,72 & 11,74 \\
\hline Média & 100,00 & 12,96 & 92,09 & 10,36 & 149,68 & 28,68 & 667,08 & 42,86 \\
\hline
\end{tabular}

Em relação a qualidade das soluções, em termos de limitante superior, a heurística HEUR-I se mostrou competitiva ao CPLEX. Note que em geral, a heurística HEUR-I encontrou limitantes superiores $1 \%$ melhores em relação aos valores obtidos pelo CPLEX. Para as instâncias com capacidade de 110, 120 e 130, a HEUR-I melhorou significativamente as soluções sendo 12,44\%, $23,18 \%$ e $68,82 \%$, respectivamente, melhores que as obtidas pelo CPLEX.

A Tabela 10 mostra que o CPLEX e as heurísticas obtiveram tempos computacionais próximos ao limite fixado para estas instâncias (1500 segundos).

As Figuras 4a e 4b mostram os desempenhos dos métodos por meio de perfis de desempenho.

Nota-se na Figura 4a que a heurística HEUR-I e o CPLEX têm os melhores desempenhos com aproximadamente $70 \%$ e $40 \%$ de chances de encontrarem os melhores valores para a função objetivo (quando $\tau=1$ ). As heurísticas HEUR-II e HEUR-III têm os piores desempenhos. Em relação aos tempos gastos, pode-se dizer que as heurísticas HEUR-I, HEUR-II e HEUR-III foram significantemente mais rápidas que o CPLEX.

Em termos práticos, esses resultados mostram que para as instâncias em que o CPLEX teve mais dificuldade em achar bons limitantes, a heurística HEUR-I, em particular, atingiu bons resultados tanto em relação aos tempos computacionais quanto em relação à qualidade das soluções. 
Tabela 10: Tempos gastos para as instâncias com 24 itens, 24 máquinas e 15 períodos.

\begin{tabular}{rrrrr}
\hline Cap & CPLEX & HEUR-I & HEUR-II & HEUR-III \\
\hline 40 & 1418,89 & $\mathbf{7 8 , 7 7}$ & 682,67 & 441,74 \\
50 & 1500,03 & $\mathbf{3 9 8 , 9 6}$ & 1035,52 & 693,96 \\
60 & 1500,03 & $\mathbf{5 4 7 , 9 6}$ & 1073,39 & 837,32 \\
70 & 1500,03 & $\mathbf{9 3 6 , 0 7}$ & 1084,61 & 1080,90 \\
80 & 1500,03 & $\mathbf{1 1 1 4 , 8 4}$ & 1136,63 & 1141,77 \\
90 & 1500,03 & 1304,46 & 1228,73 & $\mathbf{1 1 7 8 , 7 3}$ \\
100 & 1500,03 & 1340,00 & $\mathbf{1 1 9 0 , 5 6}$ & 1219,90 \\
110 & 1500,03 & 1430,22 & $\mathbf{1 2 2 4 , 6 3}$ & 1373,63 \\
120 & 1500,03 & 1435,57 & $\mathbf{1 0 7 0 , 4 5}$ & 1394,61 \\
130 & 1500,03 & 1440,86 & $\mathbf{9 7 3 , 8 6}$ & 1418,86 \\
140 & 1500,03 & 1423,38 & 1166,45 & $\mathbf{8 4 5 , 6 3}$ \\
150 & 1500,03 & 1425,42 & 1344,13 & $\mathbf{2 4 1 , 2 5}$ \\
\hline Média & 1493,27 & 1073,04 & 1100,97 & $\mathbf{9 8 9 , 0 2}$ \\
\hline
\end{tabular}

Figura 4: Perfil de desempenho dos modelos e CPLEX para encontrar solução para as instâncias com 24 itens e 24 máquinas.

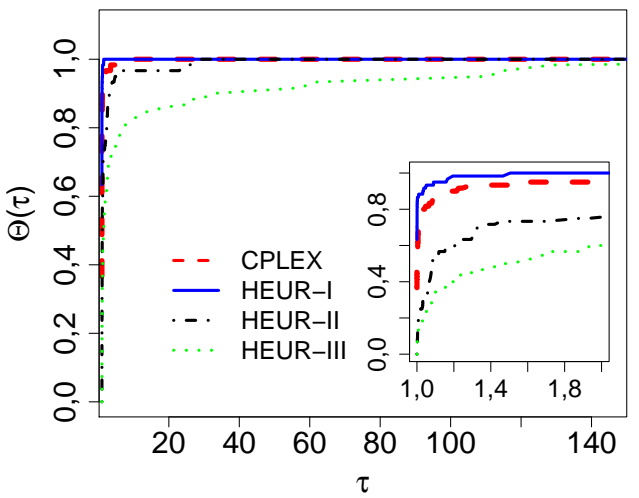

(a) Perfil de desempenho em relação ao valor da função objetivo.

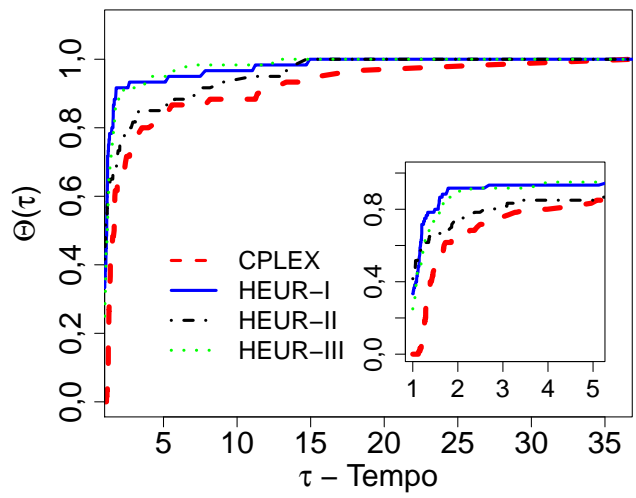

(b) Perfil de desempenho em relação ao tempo.

\section{CONCLUSÕES E PROPOSTAS FUTURAS}

Neste trabalho foi estudado um problema de dimensionamentos de lotes com máquinas paralelas, com enfoque no caso em que as máquinas são flexíveis e possuem restrições de capacidade, ou seja, cada uma das máquinas pode ou não ser flexíveis para produzir cada um dos itens.

A pesquisa baseou-se em Fiorotto et al. [4], que apresentam a formulação (2.1) - (2.9), para o problema de dimensionamento de lotes com máquinas paralelas flexíveis e que considera a 
possibilidade de investir na flexibilidade das máquinas. Em Fiorotto et al. [4] os autores relatam que, para instâncias com mais de 12 itens e 12 máquinas e capacidade média de 100 a 130, obter boas soluções para o problema é uma tarefa difícil. Sendo assim, o objetivo desse trabalho foi desenvolver três heurísticas para encontrar soluções de boa qualidade para esse problema, em baixos tempos computacionais.

Após a implementação das estratégias foram realizados os testes computacionais, com a utilização do solver CPLEX v. 12.9. Os testes foram realizados para 360 instâncias conhecidas da literatura. Nas análises consideraram-se instâncias de diferentes tamanhos e diferentes níveis de capacidade. As soluções foram analisadas através dos limitantes superiores, os tempos computacionais e o tamanho do gap entre as soluções encontradas pelos métodos e o limitante inferior obtido pelo CPLEX.

A partir dos resultados obtidos conclui-se que conforme aumenta-se o número de itens e de máquinas, devido ao aumento no tamanho do problema, o CPLEX tem dificuldades em encontrar boas soluções. A heurística HEUR-I, pelo fato de resolver problemas menores a cada iteração, foi a que obteve os melhores resultados, principalmente para problemas com 12 e 24 itens e com nível de capacidade entre 100 e 130. Em geral, o tempo computacional de todas as heurísticas é inferior ao tempo computacional gasto pelo CPLEX.

Tendo em vista estender o trabalho, bem como melhorar os resultados computacionais sugerese: melhorar as heurísticas e estudar problemas não balanceados em que o número de itens e máquinas são diferentes. A utilização de uma heurística de melhoria é uma opção para melhorar as heurísticas propostas. Além disso, para acelerar a busca pela solução através do solver é possível usar as soluções encontradas pelas heurísticas como ponto de partida.

\title{
AGRADECIMENTOS
}

Este trabalho teve o apoio financeiro da CAPES, CNPq (processos números 305261/2018-5, 406335/2018-4 e 405048/2018-1 ) e FAPESP (processos números 2013/07375-0, 2018/18754-5, 2016/01860-1 e 2019/00614-5).

\begin{abstract}
This work is related to the lot sizing problem with flexible parallel machines, which basically consists in determining the quantity of items to be produced, on each of the machines, in a finite time horizon, satisfying a given demand. This problem has an economic origin and involves costs of production, inventory and setup. In the standard problem, each item can be produced on any of the machines, i.e., we have the total machine flexibility. However, it is not always feasible to have total machine flexibility due to the costs. Therefore, it may be important to implement only a limited amount of flexibility. The investment of upgrading a machine to produce a specific product becomes a binary decision variable and there is a global budget on investment decisions. A mathematical formulation to this problem was proposed in the literature and the computational results obtained throwgh a combinatorial optimization package showed that solve the formulation is very difficult, especially for instances with many items. So in this work we have proposed three different
\end{abstract}


heuristics for this problem, in order to find good solutions in low computational times. Experiments were performed to compare the heuristics with the resolution of the model via optimization package. We have seen that by increasing the number of items and machines, the results with better quality were obtained by only one of the heuristics. In terms of computational times, all the heuristics achieved inferior times in average over CPLEX.

Keywords: lot sizing problem, machine flexibility, heuristics.

\section{REFERÊNCIAS}

[1] S. Andradóttir, H. Ayhan \& D.G. Down. Design principles for flexible systems. Production and Operations Management, 22(5) (2013), 1144-1156.

[2] E.D. Dolan \& J.J. Moré. Benchmarking optimization software with performance profiles. Math. Program., Ser. A, 91 (2002), 201-213.

[3] C.H. Fine \& R.M. Freund. Optimal Investment in Product-Flexible Manufacturing Capacity. Management Science, 36(4) (1990), 449-466. doi:10.1287/mnsc.36.4.449.

[4] D.J. Fiorotto, R. Jans \& S.A. de Araujo. Process flexibility and the chaining principle in lot sizing problems. International Journal of Production Economics, 204 (2018), 244-263. doi:10.1016/j.jpe. 2018.07.030.

[5] S.C. Graves \& B.T. Tomlin. Process Flexibility in Supply Chains. Management Science, 49(7) (2003), 907-919. doi:10.1287/mnsc.49.7.907.16381.

[6] S. Gurumurthi \& S. Benjaafar. Modeling and analysis of flexible queueing systems. Naval Research Logistics, 51(5) (2004), 755-782. doi:10.1002/nav.20020.

[7] J.P. Ignizio. Cycle time reduction via machine-to-operation qualification. International Journal of Production Research, 47(24) (2009), 6899-6906.

[8] R.R. Inman, W.C. Jordan \& D.E. Blumenfeld. Chained cross-training of assembly line workers. International Journal of Production Research, 42(10) (2004), 1899-1910. doi:10.1080/ 00207540410001666224 .

[9] S.M. Iravani, M.P.V. Oyen \& K.T. Sims. Structural Flexibility: A New Perspective on the Design of Manufacturing and Service Operations. Management Science, 51(2) (2005), 151-166. doi:10.1287/ mnsc.1040.0333.

[10] R. Jans \& Z. Degraeve. Improved lower bounds for the capacitated lot sizing problem with setup times. Operations Research Letters, 32(2) (2004), 185-195. doi:10.1016/j.orl.2003.06.001.

[11] C. Johnzèn, S. Dauzère-Pérès \& P. Vialletelle. Flexibility measures for qualification management in wafer fabs. Production Planning \& Control, 22(1) (2011), 81-90. doi:10.1080/09537287.2010. 490022.

[12] W.C. Jordan \& S.C. Graves. Principles on the Benefits of Manufacturing Process Flexibility. Management Science, 41(4) (1995), 577-594. doi:10.1287/mnsc.41.4.577. 
[13] H.Y. Mak \& Z.J.M. Shen. Stochastic programming approach to process flexibility design. Flexible Services and Manufacturing Journal, 21(3-4) (2009), 75-91. doi:10.1007/s10696-010-9062-3.

[14] A. Muriel, A. Somasundaram \& Y. Zhang. Impact of Partial Manufacturing Flexibility on Production Variability. Manufacturing \& Service Operations Management, 8(2) (2006), 192-205. doi:10.1287/ msom.1060.0099.

[15] Y. Pochet \& L.A. Wolsey. "Production planning by mixed integer programming”. Springer Science \& Business Media (2006).

[16] M. Rowshannahad, S. Dauzere-Peres \& B. Cassini. Capacitated qualification management in semiconductor manufacturing. Omega, 54 (2015), 50-59.

[17] M. Sheikhzadeh, S. Benjaafar \& D. Gupta. Machine sharing in manufacturing systems: Total flexibility versus chaining. International Journal of Flexible Manufacturing Systems, 10(4) (1998), $351-378$.

[18] D. Simchi-Levi \& Y. Wei. Understanding the Performance of the Long Chain and Sparse Designs in Process Flexibility. Operations Research, 60(5) (2012), 1125-1141. doi:10.1287/opre.1120.1081.

[19] C.S. Tang \& E.V. Denardo. Models Arising from a Flexible Manufacturing Machine, Part I: Minimization of the Number of Tool Switches. Operations Research, 36(5) (1988), 767-777. doi:10.1287/ opre.36.5.767.

[20] W.W. Trigeiro, L.J. Thomas \& J.O. McClain. Capacitated lot sizing with setup times. Management science, 35(3) (1989), 353-366.

[21] J. Xiao, H. Yang, C. Zhang, L. Zheng \& J.N. Gupta. A hybrid Lagrangian-simulated annealingbased heuristic for the parallel-machine capacitated lot-sizing and scheduling problem with sequencedependent setup times. Computers \& Operations Research, 63 (2015), 72-82. doi:10.1016/j.cor.2015. 04.010. 